\title{
Investigating Burnout Levels And Its Determinants Among The Veterinary Medicine Students: The Case Of Istanbul University
}

\author{
๑ Nursen OZTURK ${ }^{1 *}$, $\odot$ Halil Ibrahim KILIC ${ }^{1}$, $\odot$ Bulent EKIZ ${ }^{1}$
}

1Department of Animal Breeding and Husbandry, Faculty of Veterinary Medicine, Istanbul University-Cerrahpasa, Istanbul, Turkey

Received 20-01-2021 Accepted 24-05-2021

\begin{abstract}
This study was conducted to examine burnout levels and their determinants among veterinary medicine students. Answers ( $\mathrm{n}=447$ ) to a survey formed the data of this study. Maslach Burnout Inventory - Student Scale, which was adapted to the Turkish language, was used to determine the burnout levels of the students. As a result, burnout levels were obtained as 13.31, 7.82, and 7.69 for Emotional Exhaustion (EE), Depersonalization (DP), and Personal Accomplishment (PA) components, respectively. Year of study and having a hobby affected three of the burnout components. In order to determine the relationship between students' burnout levels and students' tendency to drop out, to work in a different profession after graduation and academic failure, a logistic regression analysis was conducted. Results revealed that year of study, weighted grade point average (WGPA), EE, DP, PA, repeating academic semester(s), number of the repeated academic semester(s), and perceived difficulty of veterinary education were significantly associated with the students' intention to drop out of the faculty. Gender, living situation, monthly income, EE, DP and PA affected education-occupation mismatch. Furthermore, gender, year of study, WGPA, living situation, monthly income, EE, DP, PA, and perceived difficulty level of the veterinary education influenced repeating academic semester(s). The results of this study reveal that burnout syndrome would lead to students' dropping out of the faculty, education-occupation mismatch and academic failure. Future studies must be conducted throughout the country to assess burnout syndrome in veterinary students.

Keywords: Burnout, Veterinary medicine, Drop out, Education-occupation mismatch, Academic failure
\end{abstract}

\section{Introduction}

The veterinary profession has reported having the highest suicide records among the general population in some countries. In the USA, in a survey of 11,627 veterinarians, $17 \%$ of them had experienced suicidal ideation since leaving veterinary college. ${ }^{1}$ In the UK, 14 out of 15 studies reported that veterinarians are at risk of suicide. ${ }^{2}$ In Australia, the suicide rate for veterinarians was almost twice as high as that of the general population between 2001 2012. ${ }^{3}$ One of the possible reasons for these high suicide rates can be attributed to the high demanding characteristic of the veterinary profession ${ }^{4}$ and being predisposed to depression during veterinary education. In the UK, veterinary students were found to be more depressed than the general population. ${ }^{5}$ During veterinary education, students deal with both academic and non-academic stressors. Heavy study load, concerns about the assessment, availability of the learning materials, being a perfectionist, moving to another location, financial and social issues are the reported factors that might affect the mental health of a veterinary student. ${ }^{6,78}$ It was reported that the percentage of students with clinical depression was $32 \%$ for the firstyear veterinary faculty students. ${ }^{9}$ It is highly possible that due to the increased study load and harsh academic competition among the students, the percentage of students with clinical depression will increase until the final year if there isn't any mental support system. 
Burnout syndrome can be described as a result of being chronically exposed to stressors. Previous studies identified three dimensions of burnout to describe how people react to their jobs. ${ }^{10,11,12}$

When the three components of the syndrome, Emotional Exhaustion (EE), Depersonalization (DP), and Personal Accomplishment (PA) are met, burnout occurs. Emotional Exhaustion is described as the feeling of being drained by the continuous stressors while depersonalization is a cynical attitude towards others and personal accomplishment is being dissatisfied with oneself, lack of self-efficacy, and the tendency to evaluate oneself negatively. When burnout occurs, people suffer from increased emotional exhaustion, depersonalization, and decreased level of personal accomplishment. ${ }^{10}$ Burnout syndrome was first described by Freudenberger ${ }^{13}$ and later Maslach and Leiter ${ }^{14}$ developed one of the most used scales (Maslach Burnout Inventory-MBI) to assess the burnout levels. The scale was used for social service, healthcare, mental health, criminal justice, and education. Over the past decades, Schufelli et al., ${ }^{15}$ have adopted the MBI to assess academic burnout in students.

Burnout syndrome was frequently focused on medical students by the researchers. There are not many studies focusing on burnout for veterinary medicine at the undergraduate level. However, studies show that burnout syndrome is endemic in veterinary medicine. ${ }^{16,17}$ In order to take appropriate precautions to improve mental health and prevent suicide ideation, knowing the factors causing burnout on a veterinary faculty student has great importance.

In Turkey, burnout syndrome was studied in the veterinary profession. ${ }^{18}$ However, there is not any study that determines the prevalence and determinants of burnout syndrome in veterinary medicine students. To our knowledge, this study will be the first one to address burnout syndrome in veterinary medicine students in Turkey.

This study aims to identify the determinants of burnout syndrome and burnout levels of veterinary students. Furthermore, this study investigates whether the students' burnout scores would be a predictor of future dropouts, academic failure, and occupation-education mismatch.

\section{Materials \& Methods}

\section{Participants and Procedure}

In this study, a survey was conducted with 468 students of Istanbul University, the Faculty of Veterinary Medicine who are in their 1st, 2nd, 3rd, 4th, and 5th year. After eliminating the surveys with missing answer(s) a total of 447 students' answers were used as the material of this study. Students were asked to respond voluntarily to a written survey which was kept anonymous. Most respondents were female (52.8\%), coming from a metropolitan area (59.3\%), living with their family (37.6\%), having a monthly income of 471-1000 TL (39.6\%) and having a hobby (75.6\%) (see Table 1).

Table 1. Demographic characteristics of the respondents.

\begin{tabular}{|c|c|c|}
\hline Characteristics & $\mathbf{n}$ & $\%$ \\
\hline \multicolumn{3}{|l|}{ Gender } \\
\hline Male & 190 & 42.5 \\
\hline Female & 236 & 52.8 \\
\hline Missing & 21 & 4.7 \\
\hline \multicolumn{3}{|l|}{ Previously living in } \\
\hline Metropolitan & 265 & 59.3 \\
\hline City centre & 70 & 15.7 \\
\hline District & 59 & 13.2 \\
\hline Village & 32 & 7.2 \\
\hline Missing & 21 & 4.7 \\
\hline \multicolumn{3}{|l|}{ Year of study } \\
\hline $1^{\text {st }}$ & 84 & 18.8 \\
\hline $2^{\text {nd }}$ & 63 & 14.1 \\
\hline $3^{\text {rd }}$ & 104 & 23.3 \\
\hline $4^{\text {th }}$ & 98 & 21.9 \\
\hline $5^{\text {th }}$ & 77 & 17.2 \\
\hline Missing & 21 & 4.7 \\
\hline \multicolumn{3}{|l|}{ Living with } \\
\hline Alone & 43 & 9.6 \\
\hline With family & 168 & 37.6 \\
\hline With relatives & 16 & 3.6 \\
\hline In dormitory & 114 & 25.5 \\
\hline With friends & 85 & 19.0 \\
\hline Missing & 21 & 4.7 \\
\hline \multicolumn{3}{|l|}{ Monthly Income (TL) } \\
\hline $0-470 \mathrm{TL}$ & 115 & 25.7 \\
\hline $471-1000 \mathrm{TL}$ & 177 & 39.6 \\
\hline $1001-1500 \mathrm{TL}$ & 63 & 14.1 \\
\hline $1501+\mathrm{TL}$ & 71 & 15.9 \\
\hline Missing & 21 & 4.7 \\
\hline \multicolumn{3}{|l|}{ Having a Hobby } \\
\hline No & 88 & 19.7 \\
\hline Yes & 338 & 75.6 \\
\hline Missing & 21 & 4.7 \\
\hline
\end{tabular}

\section{Survey Design}

The survey consisted of three parts. In part I, students were-asked to give socio-demographic information, using close-ended questions. In part II, students were asked to rate their intention to drop out of the veterinary faculty, their tendency to work in a different profession after graduating from the veterinary faculty, the number of the repeated academic semester(s) if they have any, and perceived difficulty of the veterinary education. In part III, Maslach Burnout Inventory - Student Scale, ${ }^{15}$ which was adapted to the Turkish language by Çapri et al., ${ }^{19}$ was used to assess the burnout levels of the students. The Turkish version of the MBI-SS had three subscales and 13 items $(\mathrm{EE}=5$ items, $\mathrm{DP}=4$ items, and PA $=4$ items). On a Likert scale of 1 to $5(1=$ never, $2=$ sometimes, $3=$ generally, $4=$ most of the time, $5=$ always) the experienced frequency of each statement is rated in MBI-SS. The maximum scores that can be obtained for EE, DP, and PA subscales would be 25, 20, 20 while minimum scores would be 5,4 , and 4 , respectively.

\section{Statistical Analyses}

The statistical analyses were performed using SPSS 19.0 (Armonk, NY, USA). The General Linear Model was used to investigate the associations between demographics and 
burnout levels of the students. In order to identify the predictive parameters of Emotional Exhaustion, Depersonalization, and Personal Accomplishment multiple linear regression analysis with the backward elimination method was used: categorical predictors were dummy coded.

The relationship between students' demographics and their tendency to drop out of the veterinary faculty, their inclination to work in a different profession after graduating from the veterinary faculty and repeating academic semester(s) were analyzed by performing a logistic regression analysis.

\section{Results}

The mean scores of the students obtained for Emotional Exhaustion (EE), Depersonalization (DP), and Personal Accomplishment (PA) were determined as 13.31, 7.82, and 7.69 , respectively. Changes in the mean scores of EE, DP, and PA as a function of students' demographics are presented in Table 2.

The effects of the gender, previous living quarters, living situation, and monthly income on the mean scores of the $\mathrm{EE}, \mathrm{DP}$, and PA were found to be insignificant $(\mathrm{P}>0.05)$. Year of study and having a hobby had a significant effect on all of the burnout components. Students who are in their 4th-year had the highest EE (15.73) and DP (9.78) scores than students from 3rd, 2nd, and 1st-year. In addition, students without a hobby had higher EE (14.21) and DP (8.54) scores than the others. Concerning PA component, 1st-year students (8.81) and students with a hobby (8.31) showed higher scores.

Table 2. Relationship between demographics and the means of Emotional exhaustion, Depersonalization and Personal accomplishment1.

\begin{tabular}{|c|c|c|c|c|c|c|c|c|c|c|}
\hline \multirow[b]{2}{*}{ Items } & \multirow[b]{2}{*}{ Sub-groups } & \multicolumn{3}{|c|}{ EE } & \multicolumn{3}{|c|}{ DP } & \multicolumn{3}{|c|}{ PA } \\
\hline & & Mean & SE & 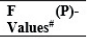 & Mean & SE & $\begin{array}{l}\mathbf{F} \\
\text { Values }^{*}\left({ }^{(P)-}-\right.\end{array}$ & Mean & SE & $\begin{array}{l}\text { Falues } \\
\text { Ve }^{*}(\mathbf{P})-\end{array}$ \\
\hline Gender & $\begin{array}{l}\text { Male } \\
\text { Female }\end{array}$ & $\begin{array}{l}13.19 \\
13.43\end{array}$ & $\begin{array}{l}0.44 \\
0.50\end{array}$ & $0.24(0.626)$ & $\begin{array}{l}8.21 \\
7.44\end{array}$ & $\begin{array}{l}0.37 \\
0.43\end{array}$ & $\begin{array}{l}3.52 \\
(0.061)\end{array}$ & $\begin{array}{l}7.78 \\
7.60\end{array}$ & $\begin{array}{l}0.27 \\
0.31\end{array}$ & $0.39(0.533)$ \\
\hline \multirow[t]{2}{*}{$\begin{array}{l}\text { Previously } \\
\text { living in }\end{array}$} & Metropolitan & 13.70 & 0.43 & & 8.49 & 0.37 & \multirow[b]{2}{*}{$\begin{array}{l}1.78 \\
(0.151)\end{array}$} & 7.28 & 0.26 & \multirow[b]{2}{*}{$2.25(0.082)$} \\
\hline & $\begin{array}{l}\text { City center } \\
\text { District } \\
\text { Village }\end{array}$ & $\begin{array}{l}13.31 \\
13.00 \\
13.24\end{array}$ & $\begin{array}{l}0.64 \\
0.69 \\
0.87\end{array}$ & $0.37(0.773)$ & $\begin{array}{l}7.26 \\
7.67 \\
7.87 \\
\end{array}$ & $\begin{array}{l}0.54 \\
0.58 \\
0.74\end{array}$ & & $\begin{array}{l}8.33 \\
7.60 \\
7.55 \\
\end{array}$ & $\begin{array}{l}0.39 \\
0.42 \\
0.53\end{array}$ & \\
\hline Year of study & $\begin{array}{l}1^{\text {th }} \\
2^{\text {nat }} \\
3^{\text {rid }} \\
4^{\text {th }} \\
5^{\text {th }} \\
\end{array}$ & $\begin{array}{l}10.41^{\mathrm{cd}} \\
11.40^{\mathrm{c}} \\
14.12^{\mathrm{b}} \\
15.73^{\mathrm{a}} \\
14.89^{\mathrm{ab}}\end{array}$ & $\begin{array}{l}0.64 \\
0.69 \\
0.56 \\
0.57 \\
0.61\end{array}$ & $\begin{array}{l}18.84 \\
(<0.001)\end{array}$ & $\begin{array}{l}4.38^{\mathrm{d}} \\
6.5^{\mathrm{c}} \\
8.6^{\mathrm{b}} \\
9.7^{\mathrm{a}} \\
9.72^{\mathrm{ab}}\end{array}$ & $\begin{array}{l}0.54 \\
0.58 \\
0.48 \\
0.49 \\
0.52\end{array}$ & $\begin{array}{l}26.49 \\
(<0.001)\end{array}$ & $\begin{array}{l}.1 .8^{2} \\
7.43^{\mathrm{b}} \\
7.2^{\mathrm{b}^{\mathrm{b}}} \\
7.42^{\mathrm{b}} \\
7.55^{\mathrm{b}}\end{array}$ & $\begin{array}{l}0.39 \\
0.42 \\
0.34 \\
0.35 \\
0.37\end{array}$ & $\begin{array}{l}3.93 \\
(0.004)\end{array}$ \\
\hline \multirow[t]{2}{*}{ Living situation } & Alone & 12.85 & 0.78 & & 8.06 & 0.66 & \multirow[b]{2}{*}{$\begin{array}{l}0.35 \\
(0.842)\end{array}$} & 7.96 & 0.47 & \multirow[b]{2}{*}{$1.71(0.148)$} \\
\hline & $\begin{array}{l}\text { With family } \\
\text { With relative(s) } \\
\text { In dormitory } \\
\text { With friend(s) }\end{array}$ & $\begin{array}{l}13.08 \\
14.47 \\
12.76 \\
13.40\end{array}$ & $\begin{array}{l}0.55 \\
1.19 \\
0.53 \\
0.59\end{array}$ & $0.58(0.675)$ & $\begin{array}{l}7.37 \\
8.04 \\
7.74 \\
7.90 \\
\end{array}$ & $\begin{array}{l}0.47 \\
1.01 \\
0.45 \\
0.50\end{array}$ & & $\begin{array}{l}8.08 \\
7.82 \\
7.66 \\
6.94\end{array}$ & $\begin{array}{l}0.34 \\
0.72 \\
0.32 \\
0.36\end{array}$ & \\
\hline \multirow[t]{2}{*}{$\begin{array}{l}\text { Monthly } \\
\text { Income (TL) }\end{array}$} & $0-470$ (IG I) & 12.88 & 0.56 & & 7.10 & 0.47 & \multirow[b]{2}{*}{$\begin{array}{l}2.31 \\
(0.075)\end{array}$} & 7.85 & 0.34 & \multirow[b]{2}{*}{$0.23(0.874)$} \\
\hline & $\begin{array}{l}471-1000 \text { (IG-II) } \\
10001-1500 \text { (GG--II) } \\
1501+\text { (IG-IV) }\end{array}$ & $\begin{array}{l}13.09 \\
14.10 \\
13.17\end{array}$ & $\begin{array}{l}0.49 \\
0.70 \\
0.64\end{array}$ & $0.84(0.471)$ & $\begin{array}{l}7.50 \\
8.01 \\
8.67\end{array}$ & $\begin{array}{l}0.41 \\
0.60 \\
0.54\end{array}$ & & $\begin{array}{r}7.71 \\
7.45 \\
7.75 \\
\end{array}$ & $\begin{array}{l}0.30 \\
0.43 \\
0.39\end{array}$ & \\
\hline $\begin{array}{l}\text { Having } \\
\text { Hobby }\end{array}$ & $\begin{array}{l}\text { No } \\
\text { Yes }\end{array}$ & $\begin{array}{l}14.21 \\
12.42\end{array}$ & $\begin{array}{l}0.58 \\
0.39\end{array}$ & $\begin{array}{l}9.70 \\
(0.002)\end{array}$ & $\begin{array}{l}8.54 \\
7.10\end{array}$ & $\begin{array}{l}0.50 \\
0.33\end{array}$ & $\begin{array}{l}8.71 \\
(0.003)\end{array}$ & $\begin{array}{l}7.07 \\
8.31\end{array}$ & $\begin{array}{l}0.35 \\
0.24\end{array}$ & $\begin{array}{l}12.59 \\
(<0.001)\end{array}$ \\
\hline Overall mean & & 13.31 & 0.40 & & 7.82 & 0.34 & & 7.69 & 0.25 & \\
\hline
\end{tabular}

\# F and P values obtained by GLM analysis. ${ }^{a, b, c, d}$ Means in the same column with different superscripts are significantly different.

1 EE: Emotional exhaustion, DP: Depersonalization and PA: Personal accomplishment

The results of the regression analysis showed that 4th-year students, students from the IG-III group, and students without a hobby ended up with an increase in the scores of
EE $(\mathrm{P}<0.001)$ while 1 st and 2 nd -year students had lower scores. Males, students coming from a metropolitan area, and students without a hobby scored higher for DP whereas 1st, 2nd, 3rd -year students, and students from IG-I and IG-II groups scored lower $(\mathrm{P}<0.001)$. It was observed that students previously living in city centers, 1st-year students, students living with their family had higher scores on PA while students without a hobby scored lower $(\mathrm{P}<0.001)$

The results of the logistic regression analysis show the predictors of students' intention to drop out of the Veterinary faculty in Table 4 . According to the results, 1st-year students, students having higher WGPA, and higher PA

Table 3. Multiple linear regression estimation of the relationships between demographics and the means of Emotional Exhaustion, Depersonalization and Personal Accomplishment1.

\begin{tabular}{|c|c|c|c|c|c|c|}
\hline & Parameter & B & SE & B & t & $\mathbf{R}^{2}$ \\
\hline \multirow{5}{*}{$\mathrm{EE}$} & $1^{\text {st }}$ year & -4.11 & 0.61 & -0.32 & -6.70 & \multirow{5}{*}{0.200} \\
\hline & $2^{\text {nd }}$ year & -2.95 & 0.67 & -0.21 & -4.43 & \\
\hline & $4^{\text {th }}$ year & 1.35 & 0.57 & 0.11 & 2.37 & \\
\hline & IG-III & 1.08 & 0.63 & 0.08 & 1.72 & \\
\hline & No hobby & 1.70 & 0.55 & 0.14 & 3.07 & \\
\hline \multirow{8}{*}{ DP } & Male & 0.85 & 0.39 & 0.09 & 2.17 & \multirow{8}{*}{0.278} \\
\hline & Metropolitan & 0.80 & 0.40 & 0.09 & 1.99 & \\
\hline & $1^{\text {st }}$ year & -5.38 & 0.52 & -0.48 & 10.32 & \\
\hline & $2^{\text {nd }}$ year & -3.23 & 0.58 & -0.26 & -5.60 & \\
\hline & $3^{\text {rd }}$ year & -1.12 & 0.48 & -0.11 & -2.32 & \\
\hline & IG-I & -1.40 & 0.51 & -0.14 & -2.77 & \\
\hline & IG-II & -0.89 & 0.45 & -0.10 & -2.01 & \\
\hline & No hobby & 1.52 & 0.48 & 0.14 & 3.20 & \\
\hline \multirow{4}{*}{ PA } & City center & 0.90 & 0.37 & 0.12 & 2.41 & \multirow{4}{*}{0.107} \\
\hline & $1^{\text {st }}$ year & 1.57 & 0.34 & 0.22 & 4.61 & \\
\hline & $\begin{array}{l}\text { Living with } \\
\text { family }\end{array}$ & 0.56 & 0.29 & 0.09 & 1.95 & \\
\hline & No hobby & -1.19 & 0.34 & -0.17 & -3.51 & \\
\hline
\end{tabular}

scores are less likely to drop out. However, students who scored higher for EE, DP, students repeating academic semester(s), and students with a higher perceived difficulty

Table4. Logistic regression estimation of the relationships between demographics and students' intention to drop out of the Veterinary Faculty

\begin{tabular}{|c|c|c|c|c|c|c|c|c|}
\hline \multirow[t]{2}{*}{ Parameter } & \multirow[t]{2}{*}{ B } & \multirow[t]{2}{*}{$\mathrm{SE}$} & \multirow[t]{2}{*}{ Wald } & \multirow[t]{2}{*}{ Sig. } & \multirow[t]{2}{*}{$\operatorname{Exp}(\mathbf{B})$} & \multicolumn{2}{|c|}{$\begin{array}{l}95 \% \text { C.I. for } \\
\text { EXP(B) }\end{array}$} & \multirow{2}{*}{$\begin{array}{c}\text { Nagelkerke } \\
\mathbf{R} \\
\text { square }\end{array}$} \\
\hline & & & & & & & Upper & \\
\hline Gender & & & & & & & & \\
\hline \multirow{2}{*}{\multicolumn{9}{|c|}{$\begin{array}{l}\text { Previously living in } \\
\text { Pold }\end{array}$}} \\
\hline & & & & & & & & \\
\hline Metropolitan & -0.24 & 0.38 & 0.38 & 0.538 & 0.79 & 0.37 & 1.67 & 0.006 \\
\hline City center & 0.09 & 0.43 & 0.05 & 0.832 & 1.10 & 0.47 & 2.56 & \\
\hline \multirow{2}{*}{\multicolumn{9}{|c|}{$\begin{array}{l}\text { District } \\
\text { Year of study }\end{array}$}} \\
\hline & & & & & & & & \\
\hline $1^{\text {st }}$ year & -1.13 & 0.37 & 9.47 & 0.002 & 0.32 & 0.16 & 0.66 & 0.067 \\
\hline $\begin{array}{l}2^{\text {nd }} \text { year } \\
\text { nat }\end{array}$ & $\begin{array}{l}-0.37 \\
-1.37 \\
Y\end{array}$ & 0.36 & 1.08 & 0.299 & 0.69 & 0.34 & 1.39 & \\
\hline $\begin{array}{l}3^{\text {rd }} \text { year } \\
\text { year }\end{array}$ & 0.28 & 0.30 & 0.84 & 0.359 & 1.32 & 0.73 & 2.40 & \\
\hline $4^{\text {th }}$ year & 0.07 & 0.31 & 0.04 & 0.833 & 1.07 & 0.58 & 1.96 & \\
\hline WGPA & -0.78 & 0.19 & 16.04 & $<0.001$ & 0.46 & 0.32 & 0.67 & 0.053 \\
\hline \multicolumn{9}{|l|}{ Living with } \\
\hline Alone & -0.12 & 0.39 & 0.09 & 0.760 & 0.89 & 0.42 & 1.89 & \\
\hline With family & -0.45 & 0.28 & 2.65 & 0.104 & 0.64 & 0.37 & 1.10 & \\
\hline With relative(s) & 0.41 & 0.55 & 0.55 & 0.458 & 1.50 & 0.51 & 4.38 & \\
\hline \multirow{2}{*}{\multicolumn{9}{|c|}{$\begin{array}{c}\text { In dormitory } \\
\text { Monthly Income (TL) }\end{array}$}} \\
\hline & & & & & & & & \\
\hline $0-470$ (IG I) & 0.32 & 0.32 & 1.01 & 0.315 & 1.38 & 0.74 & 2.59 & 0.007 \\
\hline 471-1000 (IG-II) & 0.21 & 0.30 & 0.48 & 0.490 & 1.23 & 0.68 & 2.20 & \\
\hline 1001-1500 (IG-III) & 0.51 & 0.36 & 2.02 & 0.156 & 1.67 & 0.82 & 3.39 & \\
\hline Having a Hobby & 0.35 & 0.24 & 2.05 & 0.153 & 1.42 & 0.88 & 2.28 & 0.006 \\
\hline $\mathrm{EE}$ & 0.20 & 0.03 & 64.35 & $<0.001$ & 1.23 & 1.23 & 1.17 & 0.237 \\
\hline DP & 0.16 & 0.03 & 43.65 & $<0.001$ & 1.18 & 1.21 & 1.23 & 0.147 \\
\hline & -0.23 & 0.04 & 33.60 & $<0.001$ & 0.79 & 0.73 & 0.86 & 0.120 \\
\hline \multirow{3}{*}{$\begin{array}{l}\text { Repeating academic semester(s) } \\
\text { Number of repeating academic } \\
\text { semester(s) } \\
\text { The perceived diffficulty level of the }\end{array}$} & 0.67 & 0.23 & 8.23 & 0.004 & 1.96 & 1.24 & 3.09 & 0.026 \\
\hline & 0.42 & 0.11 & 15.71 & $<0.001$ & 1.52 & 1.24 & 1.87 & 0.054 \\
\hline & 0.49 & 0.18 & 7.73 & 0.005 & 1.63 & 1.16 & 2.31 & 0.027 \\
\hline
\end{tabular}

1 EE: Emotional exhaustion, DP: Depersonalization and PA: Personal accomplishment 
level of veterinary education are prone to leave higher education.

The determinants of students' tendency to work in a different profession after their graduation are shown in Table 5. Male students and students with higher EE and DP scores mostly tend to work in a different profession while Table 5. Logistic regression estimation of the relationships between demographics and students' tendency to work in a different profession

\begin{tabular}{|c|c|c|c|c|c|c|c|c|}
\hline \multirow{2}{*}{ Parameter } & \multirow[t]{2}{*}{ B } & \multirow[t]{2}{*}{$\mathbf{S E}$} & \multirow{2}{*}{ Wald } & \multirow{2}{*}{ sig. } & \multirow{2}{*}{ Exp(B) } & \multicolumn{2}{|c|}{$95 \%$ C.I. for EXP(B) } & \multirow{2}{*}{$\begin{array}{c}\text { Nagelkerke } \mathbf{R} \\
\text { square }\end{array}$} \\
\hline & & & & & & Lower & Upper & \\
\hline Male & 0.82 & & & $<0,001$ & & & & \\
\hline \multicolumn{9}{|l|}{$\begin{array}{l}\text { Male } \\
\text { Previously living in }\end{array}$} \\
\hline Metropolitan & -0.72 & 0.40 & 3.17 & 0.075 & 0.49 & 0.22 & 1.08 & 0.016 \\
\hline City centre & -0.27 & 0.46 & 0.35 & 0.555 & 0.76 & 0.31 & 1.87 & \\
\hline District & -0.62 & 0.49 & 1.61 & 0.205 & 0.54 & 0.21 & 1.40 & \\
\hline \multicolumn{9}{|l|}{ Year of study } \\
\hline $1^{ \pm}$year & -0.35 & 0.40 & 0.75 & 0.386 & 0.71 & 0.32 & 1.55 & 0.010 \\
\hline $2^{\text {du year }}$ & 0.18 & 0.40 & 0.21 & 0.646 & 1.20 & 0.55 & 2.63 & \\
\hline $3^{\text {th year }}$ & 0.21 & 0.35 & 0.36 & 0.547 & 1.24 & 0.62 & 2.48 & \\
\hline $4^{\star m}$ year & 0.14 & 0.36 & 0.14 & 0.709 & 1.15 & 0.56 & 2.33 & \\
\hline WGPA & -0.38 & 0.21 & 3.19 & 0.074 & 0.69 & 0.45 & 1.04 & 0.011 \\
\hline \multicolumn{9}{|l|}{ Living with } \\
\hline Alone & -0.25 & 0.42 & 0.35 & 0.556 & 0.78 & 0.34 & 1.78 & 0.018 \\
\hline Family & -0.63 & 0.31 & 4.19 & 0.041 & 0.53 & 0.29 & 0.97 & \\
\hline Relative(s) & 0.03 & 0.59 & 0.00 & 0.958 & 1.03 & 0.33 & 3.27 & \\
\hline \multirow{2}{*}{\multicolumn{9}{|c|}{ Mouthly Income (TL) }} \\
\hline & & & & & & & & \\
\hline 0-470 (IG I) & -0.76 & 0.35 & 4.75 & 0.029 & 0.47 & 0.24 & 0.93 & 0.017 \\
\hline 471-1000 (IG-II) & -0.53 & 0.31 & 2.87 & 0.090 & 0.59 & 0.32 & 1.09 & \\
\hline $1001-1500$ (IG-III) & -0.43 & 0.39 & 1.20 & 0.273 & 0.65 & 0.30 & 1.40 & \\
\hline Having a Hobby & 0.51 & 0.27 & 3.64 & 0.056 & 1.67 & 0.99 & 2.81 & 0.012 \\
\hline & 0.11 & 0.03 & 18.11 & $<0.001$ & 1.11 & 1.06 & 1.17 & 0.069 \\
\hline DP & 0.14 & 0.03 & 27.79 & $<0.001$ & 1.15 & 1.09 & 1.21 & 0.101 \\
\hline & -0.16 & 0.04 & 13.38 & $<0.001$ & 0.86 & 0.79 & 0.93 & 0.050 \\
\hline Repeating academic semester(s) & -0.20 & 0.28 & 0.48 & 0.487 & 0.82 & 0.47 & 1.43 & 0.002 \\
\hline Number of repeating academic semester(s) & 0.10 & 0.11 & 0.84 & 0.359 & 1.11 & 0.89 & 1.37 & 0.003 \\
\hline Perceived difficulty of the veterinary edication & -0.02 & 0.18 & 0.01 & 0.928 & 0.98 & 0.70 & 1.39 & 0.000 \\
\hline
\end{tabular}

students living with their family, from the lowest monthly income and higher PA scores were less likely to work in a different profession other than Veterinary medicine. Furthermore, students having lower WGPA (Weighted Grade Point Average $)(\mathrm{P}=0.074)$ and students with a hobby $(\mathrm{P}=$ $0.056)$ showed a tendency to work in a different profession. The results of the logistic regression analysis revealed that male students with high scores in EE, DP, and perceived difficulty level of veterinary education tend to fail semester(s). On the other hand, 1st year students, students with high WGPA, students living with their family and living in a dormitory, and students who are from the IG-I and IG-II are less likely to fail (Table 6.)

\section{Discussion}

In this study, burnout levels and their determinants were assessed. Year of study and having a hobby are the main effects on students' burnout scores. Students in their 4th Table 6. Logistic regression estimation of the relationships between demographics and repeating academic semester(s)

\begin{tabular}{|c|c|c|c|c|c|c|c|c|}
\hline \multirow[t]{2}{*}{ Parameter } & \multirow[t]{2}{*}{ B } & \multirow[t]{2}{*}{ SE } & \multirow[t]{2}{*}{ Wald } & \multirow[t]{2}{*}{ Sig. } & \multirow[t]{2}{*}{$\operatorname{Exp}(B)$} & \multicolumn{2}{|c|}{$\begin{array}{l}95 \% \text { C.I. for } \\
\text { EXP(B) }\end{array}$} & \multirow{2}{*}{$\begin{array}{c}\text { Nagelkerke } \\
\mathbf{R} \\
\text { square } \\
\end{array}$} \\
\hline & & & & & & & Upper & \\
\hline $\begin{array}{l}\text { Gender } \\
\end{array}$ & & & & & & & & \\
\hline & 0.44 & 0.23 & 3.66 & 0.056 & 1.56 & 0.99 & 2.45 & 0.013 \\
\hline \multicolumn{8}{|l|}{$\begin{array}{l}\text { Male } \\
\text { Previously living in }\end{array}$} & \multirow{4}{*}{0.007} \\
\hline Metropolitan & -0.48 & 0.41 & 1.40 & 0.237 & 0.62 & 0.28 & 1.38 & \\
\hline City center & -0.27 & 0.47 & 0.34 & 0.562 & 0.76 & 0.30 & 1.91 & \\
\hline District & -0.58 & 0.50 & 1.33 & 0.249 & 0.56 & 0.21 & 1.50 & \\
\hline \multicolumn{9}{|l|}{ Year of study } \\
\hline $1^{\text {ts }^{t} \text { year }}$ & -1.91 & 0.52 & 13.23 & $<0.001$ & 0.15 & 0.05 & 0.42 & \multirow{4}{*}{0.078} \\
\hline $2^{\text {nd }}$ year & -0.31 & 0.39 & 0.64 & 0.423 & 0.73 & 0.34 & 1.57 & \\
\hline $3^{\text {rd }}$ year & -0.25 & 0.34 & 0.53 & 0.466 & 0.78 & 0.41 & 1.51 & \\
\hline $4^{\text {th }}$ year & -0.01 & 0.33 & 0.00 & 0.968 & 0.99 & 0.51 & 1.90 & \\
\hline WGPA & -2.35 & 0.30 & 61.67 & $<0.001$ & 0.10 & 0.05 & 0.17 & 0.066 \\
\hline \multicolumn{9}{|l|}{ Living with } \\
\hline Alone & -0.07 & 0.39 & 0.03 & 0.860 & 0.93 & 0.43 & 2.01 & \multirow[t]{3}{*}{0.062} \\
\hline With family & -0.93 & 0.30 & 9.60 & 0.002 & 0.39 & 0.22 & 0.71 & \\
\hline With relative(s) & -0.54 & 0.62 & 0.77 & 0.380 & 0.58 & 0.17 & 1.96 & \\
\hline \multirow{2}{*}{\multicolumn{9}{|c|}{ Monthly Income (TL) }} \\
\hline & & & & & & & & \\
\hline $\begin{array}{l}\text { 0-470 (IG I) } \\
471-1000 \text { (IG-II) }\end{array}$ & $\begin{array}{l}-1.55 \\
-1.01\end{array}$ & $\begin{array}{l}0.38 \\
0.31\end{array}$ & $\begin{array}{l}17.06 \\
10.64\end{array}$ & $\begin{array}{c}<0.001 \\
0.001\end{array}$ & $\begin{array}{l}0.21 \\
0.37\end{array}$ & $\begin{array}{l}0.10 \\
0.20\end{array}$ & $\begin{array}{l}0.44 \\
0.67\end{array}$ & \multirow[t]{2}{*}{0.084} \\
\hline $1001-1500$ (IG-III) & -0.19 & 0.36 & 0.29 & 0.590 & 0.82 & 0.41 & $\begin{array}{l}0.01 \\
1.67\end{array}$ & \\
\hline Having a Hobby & & 0.28 & 0.25 & 0.618 & & 0.67 & 1.98 & 0.001 \\
\hline $\mathrm{EE}$ & 0.08 & 0.02 & 9.81 & 0.002 & 1.08 & 1.03 & 1.13 & 0.036 \\
\hline DP & 0.08 & 0.03 & 10.80 & 0.001 & 1.09 & 1.04 & 1.14 & 0.038 \\
\hline & -0.14 & 0.04 & 11.27 & 0.001 & 0.87 & 0.80 & 0.94 & 0.042 \\
\hline The perceived difficulty level of the & 0.64 & 0.23 & 8.10 & 0.004 & 1.91 & 1.22 & 2.97 & 0.034 \\
\hline
\end{tabular}

year had higher EE and DP scores than 3rd, 2nd, and 1styear students. A heavy pre-clinical and clinical education is started on the 4th year of study and students are responsible for the clinical rotations where they start to communicate with patient owners, experiencing patients being ex and facing misdiagnosed issues. The results of this study have shown that students get affected by this work load because it is mentioned as the main contributor to stress. ${ }^{20}$ Supporting our findings, a review study that concerns medical students in China indicated that senior students suffered greater burnout. ${ }^{21}$ This has administrative implications for the faculty to consider workload that causes high burnout scores for the 4th and 5th-year students. Studies show that students develop a coping mechanism to deal with burnout syndrome. Using substances such as alcohol, drugs, and food are reported to be an unhealthy coping mechanism to get over the depressive mood. ${ }^{22}$ On the other hand, positive coping mechanisms including engaging in hobbies are effectively helpful in coping with stress. The result of this study reveals that having a hobby decreased the EE and DP scores while it increased the PA score. Student social clubs in the university enable students to establish new friendships with those from various faculties, develop social bonds and engage in their hobbies, as well as helping to build a sense of social belongingness that has an important effect on students' well being and feelings of belongingness to the university. ${ }^{5}$ Therefore, encouraging students to attend social clubs is crucial to decrease burnout levels.

Concerning the predictive values of burnout, the results showed that gender is a significant parameter for the depersonalization component. In this study, male students were found to have more cynical attitudes toward others. Studies indicate that gender affects burnout. In other words, women and men experience burnout in different ways. Purvanova and Muros ${ }^{23}$ revealed that women are slightly more exhausted than men. Men, on the other side, are more depersonalized. Income is also reported to affect burnout scores. ${ }^{24}$ The results in our study were consistent with that; a decrease in monthly income increased the DP scores of the students. Another significant predictor of burnout was the living situation of the student. Living with family increased the students' PA scores. Students who live in a supportive environment, either at university or in the community are benefiting from moral support. In the study of Chigerwe et al., ${ }^{16}$ students living with other veterinary students had a lower level of burnout.

In this study, we also assessed the predictors of students' tendency to drop out of the veterinary faculty, students' tendency to work in a different profession after graduating from veterinary faculty and to repeat academic semester(s). 
Dropping out of the faculty is an important issue that represents a human and economic loss. Experiencing financial problems, not fitting with the academic demands, suffering lack of motivation, the study not stimulating their interest, ${ }^{25}$ an increase in physical, psychological, and social problems cause students to drop out. ${ }^{26}$ In this study, the students with lower WGPA, students having higher scores on EE, DP and lower scores on PA, students repeating academic semester(s), number of the repeated academic semester(s), and students' perceived difficulty level of the veterinary education increased students' intention to drop out. Students who are chronically being exposed to stressors experience burnout, lack of self-efficacy, anxiety, and depression. Students feeling emotionally drained may not be motivated to study and eventually, this leads them to have lower WGPA scores, fail, and as a result, drop out of the faculty.

Intention to work in a different profession may be caused by education-occupation mismatch where students' interest does not match their actual study. Making an unsuitable study choice would result in a student being cynical towards the study subject and ending up in burnout. Our results showed that male students and students who are experiencing burnout intend to work in a different profession after graduation.

However, education-occupation mismatch was reported to result in income penalties. ${ }^{27}$ In a study conducted by Robts, ${ }^{28}$ it was revealed that college graduates working in a different profession earn $11 \%$ lower than their counterparts. Therefore, it is essential to match individual education skills with occupational job characteristics.

Academic failure may cause a delay in graduation and a delay in income. Furthermore, due to the increasing number of students, the lecturer-students ratio is disrupted. To protect students' and universities' financial investment, knowing the predictors of students at risk of academic failure is crucial. ${ }^{29}$ In our study, students experiencing burnout and students having a perception that veterinary education is difficult tended to fail. Furthermore, 1 st year students, students living with their family and in the dormitory, students that are from IG-I and IG-II were less likely to fail. This can be explained by the fact that first-year students are more motivated to study and have a keen interest in the veterinary subject which is consistent with their high PA scores. Also, students living with family or in dormitories probably receive emotional support from their family and friends. Considering students' monthly income, the low and middle-income group students may feel they are obligated to be successful due to their limited budget.

One limitation of this study is that, the sample was constituted by the students from Istanbul University. In order to have a solid idea of the determinants of burnout on veteri- nary students, a nation-wide survey must be implemented.

\section{Conclusion}

The results of this study reveal that students of the veterinary faculty are experiencing burnout. Furthermore, burnout scores were found to be the determinants of students' intention to drop out, education-occupation mismatch, and academic failure.

It is highly possible that students experiencing burnout during the veterinary medicine education would continue to have burnout through their professional life which would result in misdiagnosis, depression, or even a suicide decision. Therefore, faculties are recommended to consider the burnout determinants and take precautions considering these determinants while designing the curriculum to avoid possible burnouts.

It would be effective to assess burnout levels of students in each term to identify students who are dealing with burnout. Students with high burnout scores must be directed to support systems. Identifying and supporting students to deal with burnout would decrease possible dropouts, education-occupation mismatch, and academic failure.

\section{References}

1. Nett RJ, Witte TK, Holzbauer SM, Elchos BL, Campagnolo ER, Musgrave KJ. Risk factors for suicide, attitudes toward mental illness, and practice-related stressors among US veterinarians. J Am Vet Med Assoc. 2015;247(8):945-955

2. Platt B, Hawton K, Simkin S, Mellanby RJ. Systematic review of the prevalence of suicide in veterinary surgeons. Occup Med. 2010;60(6):436-446

3. Milner AJ, Niven H, Page K, LaMontagne AD. Suicide in veterinarians and veterinary nurses in Australia: 2001-2012. Aust Vet J. 2015;93(9):308-310

4. Mastenbroek NJJM, Demerouti E, Van Beukelen P, Muijtjens AMM, Scherpbier AJJA. Measuring potential predictors of burnout and engagement among young veterinary professionals; construction of a customised questionnaire (the Vet-DRQ). Vet Rec. 2014;174(7):168-168.

5. Cardwell JM, Lewis EG. Vocation, Belongingness, and Balance: A Qualitative Study of Veterinary Student Well-Being. J Vet Med Educ. 2017;44(1):29-37.

6. Moffat KJ, McConnachie A, Ross S, Morrison JM. First year medical student stress and coping in a problem-based learning medical curriculum. Med Educ. 2004;38(5):482-491.

7. Vitaliano PP, Russo J, Carr JE, Heerwagen JH. Medical school pressures and their relationship to anxiety. J Nerv Ment Dis. 1984;172(12):730-736. 
8. Bartram DJ, Baldwin DS. Veterinary surgeons and suicide: a structured review of possible influences on increased risk. Vet Rec. 2010;166(13):388-397.

9. Hafen M Jr, Reisbig AMJ, White MB, Rush BR. Predictors of depression and anxiety in the first-year veterinary students: a preliminary report. J Vet Med Educ. 2006;33(3):432-440

10. Maslach C. Burned-out. Hum. Behav. 1976; 5 (9): 1622.

11. Maslach C. The client role in staff burn-out. JSI. 1978;34(4):111-124.

12. Maslach C, Jackson SE. The measurement of experienced burnout. J. Organ. Behav. 1981;2(2):99-113.

13. Freudenberger HJ. Staff burn-out. JSI 1974;30(1):159165.

14. Maslach C, Leiter MP. The truth about burnout: How organizations cause personal stress and what to do about it. 2008.

15. Schaufeli WB, Martinez IM, Marques-Pinto A, Salanova $M$, Bakker A. Burnout and engagement in university students: A cross-national study. Cross cult Studies 2002;33(5):464-481.

16. Chigerwe M, Boudreaux KA, Ilkiw JE. Assessment of burnout in veterinary medical students using the Maslach Burnout Inventory-Educational Survey: a survey during two semesters. BMC Med Educ. 2014;14(1):255.

17. Živojinović J, Backović D, Belojević G, Valčić O, Soldatović I. Predictors of burnout among Belgrade veterinary students: A cross-sectional study. PLoS One. 2020;15(3): e0230685.

18. Babaoğlu ÜT, Cevizci S, Arslan M. İstanbul'da çalıșan Veteriner Hekimlerin iş doyumu ve tükenmişlik düzeyleri. Kafkas Univ Vet Fak Derg. 2012;18(4):599604.

19. Çapri B, Gündüz B, Gökçakan Z. Maslach Tükenmişlik Envanteri-Öğrenci Formu'nun (MTE-ÖF) Türkçe'ye Uyarlanması: Geçerlik Ve Güvenirlik Çalışması. ÇÜ Eğitim Fakültesi Dergisi. 2011; 40 (1): 134-147.

20. Collins H, Foote D. Managing stress in veterinary students. J Vet Med Educ. 2005;32(2):170-172.

21. Chunming WM, Harrison R, MacIntyre R, Travaglia J, Balasooriya C. Burnout in medical students: a systematic review of experiences in Chinese medical schools. BMC Med Educ. 2017;17(1):217.

22. Diulio AR, Dutta NM, Gauthier JM, Witte TK, Correia CJ, Angarano D. Associations among depressive symptoms, drinking motives, and risk for alcohol-related problems in veterinary students. J Vet Med Educ. 2015;42(1):11-17.

23. Purvanova RK, Muros JP. Gender differences in burn- out: A meta-analysis. J. Vocat. Behav. 2010;77(2):168185.

24. Yektatalab S, Honarmandnejad K, Janghorban R. Relationship between occupational burnout and demographic variables among nurses in Jahrom, Iran. Pan Afr Med J. 2019;34(22)

25. Pitanupong J, Sangkool J, Wiwattanaworaset P, Pongthanawisut S, Teetharathul T. Dropout thought among medical students at Faculty of Medicine Prince of Songkla University Running Head: dropout thought among medical students. J. Thammasat Univ. Med. Sch. 2020;20(2):175-184.

26. Bakker EJ, Verhaegh KJ, Kox JH, van der Beek AJ, Boot CR. Late dropout from nursing education: An interview study of nursing students' experiences and reasons. Nurse Educ Pract. 2019;39:17-25.

27. Nordin M, Persson I, Rooth DO. Education-occupation mismatch: Is there an income penalty?. Econ Educ Rev. 2010;29(6):1047-1059.

28. Robst J. Education and job match: The relatedness of college major and work Econ Educ Rev. 2007;26(4):397-407.

29. Rush B, Sanderson, MW, Elmore RG. Pre-matriculation indicators of academic difficulty during veterinary school. J Vet Med Educ. 2005;32(4):517-522. 